\title{
Research on Discipline Evaluation in Universities
}

\author{
Cheng Wang, Wenping Li, Congjun Rao \\ College of Mathematics and Computer Science, Huanggang Normal University, \\ Huanggang 438000, China \\ wangc80@163.com
}

Keywords: Discipline construction in universities; Discipline evaluation; Evaluation system; DEA

\begin{abstract}
Discipline is a carrier of the scientific research and personnel training, and the discipline level is an important indicator to measure the level and the overall strength of universities. In this paper, the practical problem of discipline evaluation in universities is studied. Concretely, a index system of discipline evaluation is presented from the aspects of human resources, financial resources, material resources, scientific research and personnel training, and then a discipline evaluation model is proposed based on the method of data envelopment analysis (DEA). This study provides a theoretical basis and method reference to quantify the level of disciplines construction in universities.
\end{abstract}

\section{Introduction}

Discipline evaluation in universities is an important content of higher education evaluation, which is a comprehensive evaluation for the purpose, value, and effect subject of talent training activities. It is also an important part of the discipline construction and management in universities, and is an urgent need to strengthen the research and attention.

Discipline evaluation in university refers to evaluate the attributes values based on a certain index and weight system in accordance with the standards of a certain discipline, and its aim to strengthen the discipline construction, ensure the quality of talents cultivation, scientific research and social services. To inspect and evaluate the discipline can help universities to sum up experience, find problems, strengthen management, promote exchange and improve works, so as to promote the reform and development of the discipline work. The significance of discipline evaluation are of three aspects. First, it is one of the important measures to improve the level of university disciplines. Quantitative level of discipline classification and assessments becomes an invisible power supervision to stimulate disciplines construction. Thus it helps to form a kind of competitive landscape in universities. Second, discipline evaluation is an important means of characteristic education of discipline in universities. There is a complete set of evaluation index system for evaluating the disciplines in universities. In order to adapt to this set of index system, universities will inevitably focus on strengthening the construction of several disciplines and professional direction, thus to form their own characteristics and advantages between disciplines. In the end, discipline evaluation is an important method of discipline management in universities. Regular discipline evaluation can exchange discipline construction experience, diagnose problems and provide the improvement measures.

For the discipline evaluation problems in universities, many experts give their views and opinions, they establish various mathematical models by strict logical reasoning and reasonable assumptions imagination. Wang [1] used the AHP method to determine the weights of evaluation index system and established a evaluation model based on the multiplication plus assessment model, and obtained a composite score formula. Chen [2] compared the discipline construction level between China and US, Britain and other countries from the main subject evaluation method of evaluation and assessment data and indicators to assess the impact etc., which provide an important evaluation reference for the discipline construction in our colleges and universities. Ren [3] presented a evaluation index system which includes human resources, material resources, scientific research, personnel training, and so on, and proposed some countermeasures to improve the performance of universities disciplines [4]. Gu [5] 
analyzed some of the problems that exist in scientific research management, and he thought that the discipline assessment plays an important role to improve the research quality in colleges and universities, and used qualitative and quantitative research methods to evaluate the discipline level. Wan [6] established a multi-level gray evaluation model and multi-level DEA algorithm to evaluate the disciplines in universities, and gave a simulation experiment to test the effectiveness of the presented models.

Based on the existing methods, we presented a index system of discipline evaluation, and then proposed a discipline evaluation model based on the method of data envelopment analysis (DEA). It tries to provide a theoretical basis and method reference to quantify the level of disciplines construction in universities.

\section{The Index System of Discipline Evaluation}

Discipline construction is a complicated system engineering, which involving the aspects of school teaching, scientific research, management, and so on. Based on the existing results in index system of discipline evaluation [7-8], here we present a index system to evaluate discipline in universities from the aspects of inputs and outputs. The detailed attributes are listed in Table 1 as follows.

Table 1. The index system to evaluate discipline in universities

\begin{tabular}{|c|c|c|}
\hline \multirow{9}{*}{ Input attributes } & Primary attributes & The secondary attributes \\
\hline & \multirow{4}{*}{ Human resources } & Number of professors \\
\hline & & Number of associate professors \\
\hline & & Number of lecturer \\
\hline & & Number of junior titles \\
\hline & \multirow{2}{*}{ Financial resources } & National special investment \\
\hline & & School self-investment \\
\hline & \multirow{2}{*}{ Material resources } & Experimental equipment \\
\hline & & Space area of teaching and research \\
\hline \multirow{12}{*}{ Output attributes } & \multirow{2}{*}{ Scientific project } & Number of national projects \\
\hline & & Number of provincial projects \\
\hline & \multirow{2}{*}{ Scientific achievements } & Number of national prize \\
\hline & & Number of provincial prize \\
\hline & \multirow{3}{*}{ Personnel training scale } & Undergraduate training scale \\
\hline & & Master training scale \\
\hline & & Doctor training scale \\
\hline & \multirow{5}{*}{ Personnel training quality } & National outstanding Ph.D. thesis \\
\hline & & Doctoral dissertation at the provincial level \\
\hline & & Master's thesis at the provincial level \\
\hline & & Bachelor's thesis at the provincial level \\
\hline & & National or provincial teaching achievement prizes \\
\hline
\end{tabular}

\section{The evaluation model}

From the attributes in Table 1, we can collect data in the aspects of input and output for a given discipline. In this section, we will give a evaluation model based on DEA. The detailed steps are as follows.

Step 1: Dimensionless treatment of data

Due to the different dimensions, we must do the dimensionless treatment, and then compute the comprehensive values of input and output.

The dimensionless treatment formula is $k_{i}=\frac{x_{i}-\min x_{i}}{\max x_{i}-\min x_{i}}, i=1,2, \cdots, n$, where $k_{i}$ is the standard values of atttributes, $x_{i}$ is the attribute values of attribute $i, \min x_{i}$ is the minimum value among the values of $x_{i}$, and max $x_{i}$ is the maximum value among the values of $x_{i}$. 
Step 2: Attribute weighting

Here we determine the weight for all attributes based on two factors. The first one is contrast intensity, which represents the value of the gap between the various evaluation program, and is in the form of standard deviation. The second is the conflict between evaluation attributes. Conflicts between attributes is based on the correlation between attributes. If there is strong positive correlation between two attributes, then there is low conflict between these two attributes.

The conflict between the $j$-th attribute and the other attributes are quantified as $\underset{n=1}{a}\left(1-r_{i j}\right)$, where $r_{i j}$ is the coefficient of association between the attribute $t$ and $j$. Let $C_{i j}$ be the information content of the $j$-th attribute, the $C_{i j}$ can be expressed by $C_{j}=S_{j} \underset{n=1}{a}\left(1-r_{i j}\right), j=1,2, \cdots, m$.

The greater the values of $C_{i j}$ is, the greater the information content of the $j$-th attribute is, which means the greater the relative importance for the $j$-th attribute. The weight of the $j$-th attribute is $W_{j}=\frac{C_{j}}{\underset{n=1}{n_{0}} C_{j}}, j=1,2, \cdots, m$.

Step 3: Compute the comprehensive values of input and output.

By the collection processing and comprehensive analysis for the values of input and output, we can get the quantity index of the DMU combined efficiency and determine whether every DMU is effective. Based on the fundamental principle of DEA, we can establish the following DEA model to evaluate the disciplines in universities.

There are many forms for the DEA model, here we use the DEA model, i.e., $C^{2} R$ to study the multiple input and multiple output decision making units, and present effective method.

Now we suppose there are $n$ DMU, and each DMU have $m$ inputs and $s$ outputs, the input and output of $D M U_{j}$ are $x_{j}=\left(x_{1 j}, x_{2 j}, \cdots, x_{m j}\right)^{T}$ and $y_{j}=\left(y_{1 j}, y_{2 j}, \cdots, y_{s j}\right)^{T}, j=1,2, \cdots, n$, which satisfy the following conditions

$$
\left\{\begin{array}{c}
\max \frac{u^{T} y_{0}}{V^{\mathrm{T}} x_{0}} \\
\text { S.T. } \frac{u^{T} y_{j}}{V^{\mathrm{T}} x_{j}} \leq 1 \\
u \geq 0, V \geq 0
\end{array}\right.
$$

which means the objective function $\frac{u^{T} y_{0}}{V^{\mathrm{T}} x_{0}}$ reaches maximum if the condition is satisfied, where $V=\left(V_{1}, V_{2}, \cdots, V_{m}\right)^{T}$ and $u=\left(u_{1}, u_{2}, \cdots, u_{s}\right)^{T}$ denote the weight coefficients of $m$ inputs and $s$ outputs. The formula (1) can be transformed into the following equivalent dual linear programming.

$$
\left\{\begin{array}{c}
\min \theta \\
\text { S.T. } \sum_{j=1}^{n} x_{j} \lambda \leq \theta x_{0} \\
\sum_{j=1}^{n} y_{j} \lambda_{j} \geq V_{0} \\
\lambda_{j} \geq 0, j=1,2, \ldots n, \theta \in E_{1}^{+}
\end{array}\right.
$$

For the convenience of solving, we can make the Archimedes infinitesimal process for (2), then we have 


$$
\left\{\begin{array}{c}
\min \left[\theta-\hat{\imath}^{\wedge T} S^{-}+e^{\wedge T} S^{+}\right] \\
\text {s.t. } \sum_{j=1}^{n} x_{j} y_{j}+S^{-}=\theta x_{0} \\
\sum_{j=1}^{n} y_{j} \lambda_{j}-S^{+}=y_{0} \\
\lambda_{j} \geq 0, j=1,2, \ldots n \\
\theta \in E_{1}^{+}, S^{-} \geq 0, S^{+} \geq 0
\end{array}\right.
$$

where $\hat{e}=(1,1, \ldots, 1)^{T}$. If the optimal solution satisfies $\theta_{0}, \lambda_{0 j} \lambda_{0 j}, j=1,2, \cdots, n$, and $S^{-}, S^{+}$satisfies $S^{-}=0, S^{+}=0 \quad \theta_{0}=1$, then $D M U_{j 0}$ is DEA effective.

\section{Conclusions}

In this paper, we discuss present research situation of discipline evaluation in universities, and present a index system of discipline evaluation from the aspects of human resources, financial resources, material resources, scientific research and personnel training. Based on this index system, we establish a discipline evaluation model via the method of DEA. In the practical evaluation, we can refer this evaluation results, and take some corresponding measures to improve the level of disciplines construction in universities. For example, clear disciplines positioning, combine the disciplinary construction with the current status of development of higher education, reasonably determine the distribution of human, financial and material resources in order to provide a solid material support for better output, strengthen the combination of industry-study-research, and so on.

\section{Acknowledgments}

This work is supported by the Natural Science Foundation of Hubei Province (No. 2015CFB420), and the Reform Project of Transformation Development of Huanggang Normal University (No. xfg2015C19).

\section{References}

[1] X.B Wang: Journal of Educational Institute of Jilin Province Vol. 29 (2013), p. 151-152.

[2] H.F. Chen: University (academic edition) Vol. 8 (2012), p. 61-67.

[3] B.B. Ren: Modern Business Trade Industry Vol. 20 (2008), p. 224-225.

[4] J. Chen: Performance Evaluation of Subject Construction in University Study (Master's degree thesis of Wuhan university of science 2010).

[5] Y. Gu: Evaluation theory and practice Vol. 29 (2007), p. 56-59.

[6] X. Wan: Corporation Research Vol. 16 (2010), p. 131-133.

[7] Y.B. Cheng: Science \& Technology Progress and Policy Vol. 9 (2008), p. 180-183.

[8] J.F. Ryan: Research in Higher Education Vol. 45 (2004), p. 97-115. 